\title{
Evidence based nursing-a crucial approach
}

\section{Introduction}

Regardless of the health care setting, the nurses provide quality care to their clients irrespective of their social, cultural or individual characteristics. The nurse initiates the care by having good interpersonal relationship with the clients and the clients are confident that the care provided by that nurse is based on the most current, up to date health information available. Thus, the need for evidence based practice (EBP) is confirmed for delivering quality nursing care.

Pravikoff, Tanner and Pierce ${ }^{1}$ describes the process of EBP as assessing, delineating a problem through verbalization of an identifiable question, pursuing and evaluating the available facts, implementing a practice intervention as a result of the evidence and evaluating the entire process for effectiveness. Initially, EBP requires the identification of the practice problem, followed by the utilization of tested results to improve the care provided for the clients.

Nurses are constantly challenged to meet the emerging and changing evidence relevant to their areas of practice. The crucial approach that empowers today's nurses to pace with evolving new literature and technological advancement to render quality care is evidence-based nursing.

The recent approach is towards self/independent learning and evidence based methods for clinical decision making as the health care practice is moving to the paradigm called "evidence based practice" As health professionals, the responsibility lies among themselves to educate appropriately and think critically in the field of health and nursing based on relevancy and quality of the evidence so as to render quality nursing care. ${ }^{2}$

Nurses spend a great deal of time in framing their preclinical objectives by designing the care plans, conceptualizing the Pathophysiology, reviewing the drug administration policy and the legal and ethical issues and concerns for clinical decision making in nursing practice. Hence they provide care based knowledge in accordance with what they learned in their nursing school and based on traditions, myths, hunches, advice of colleagues, or outdated textbooks. For example, when clinical questions arise, one may look to a nursing textbook for the answers or ask the colleague for advice which is not the right way to find the answers. Single reliance on textbooks and expert faculty knowledge does not provoke critical thinking skills and enhance clinical decision making that nurses must have to survive in the current clinical settings.

In a recent study conducted by Pravikoff DS, ${ }^{1}$ most of the nurses provide care based on what they learned from their nursing school. Also the finding is suggestive that the nurses very rarely use the research reports, journal articles and reference from hospital libraries. The findings also have a relationship with the age of the nurses which is suggestive that nurses more than 40 years the knowledge is outdated. The author concludes that nurses practicing based such knowledge does not render quality patient care.

Nursing research has paved way in the health care research enterprise to sharpen the evidence into practice in the health care history. Conducting research helps the nurses to understand how to increase effectiveness, efficiency, safety, timeliness of health care and

\author{
Volume I Issue 2 - 2016
}

Vijayalakshmi G
College of Pharmacy and Nursing, University of Nizwa, Oman

Correspondence: Vijayalakshmi G, Lecturer, College of Pharmacy and Nursing, University of Nizwa, Oman, Email gopalan@unizwa.edu.om

Received: November 07, 2016 | Published: November 21, 2016

the ways to improve health care delivery system there by rendering quality nursing care. $^{2}$

Nurses must be aware about the sources that help them to retrieve the high quality information to translate their knowledge into quality patient care. With the advancement of technology there are various types of sources that include updated textbooks, journals, online bibliographic databases, products that distill or consolidate research, and the internet which provides a critical strategy to ensure that care is up to date and that it reflects the latest research evidence.

\section{Barriers in implementing evidence-based practice}

The barriers that prevent nurses from using research in everyday practice have been cited in numerous studies and some common findings have emerged. ${ }^{3,4}$ Some of the barriers that nurses often report are: Lack of value for research in practice, difficulty in changing practice, lack of administrative support, lack of knowledgeable mentors, insufficient time to conduct research, lack of education about the research process, lack of awareness about research or evidence-based practice, difficulty accessing research reports and articles, no time on the job to read research and so on. Despite these barriers, nurses are engaging in evidence based practice and making a difference in patient outcomes. However these barriers can be overcome through the effort of the organization by focusing on integrating research in practice. Nurses can be involved in journal clubs, reviewing and critiquing research articles and integrating EBP in the nursing curriculum. The rich resources offer nursing students a chance to employing EBP and connect their emerging competencies with clinical needs for best practices in clinical settings.

\section{Opportunities and challenges}

Evidence based practice help to guide clinical management decisions. ${ }^{5}$ Clinical leaders have provided opportunity to step forward to renovate healthcare from a structural perspective on to focusing on evidence based practice for clinical effectiveness, and patient safety. The challenges for moving EBP forward are from nurses becoming powerful leaders in inter-professional groups and nurses becoming powerful influencers of change.

The nursing profession remains central to the interdisciplinary and discipline-specific changes necessary to achieve care that is effective, safe, and efficient. As practicing nurses, educators and 
administrators are we ready to take up the challenge with the support of the organization, colleagues and available resources?

\section{Conclusion}

To be successful with evidence-based practice, nurses needs to be willing to challenge their own assumptions and there by implement the most up-to-date, research-tested, and high-quality patient care. Evidence-based practice takes resources, work, time, and effort, but the outcomes make them worthwhile. Every patient deserves care that is based on the best scientific knowledge and that ensures highquality, cost-effective care.

\section{Acknowledgements}

None.

\section{Conflict of interest}

The author declares no conflict of interest.

\section{References}

1. Pravikoff DS, Tanner AB, Pierce ST. Readiness of U. S. nurses for evidence-based practice. American Journal of Nursing. 2005;105(9):40-51.

2. Stevens K. The impact of evidence-based practice in nursing and the next big ideas. Online Journal of Issues in Nursing. 2013;18(2):4.

3. Clifford C, Murray S. Pre-and post-test evaluation of a project to facilitate research development in practice in a hospital setting. Journal of Advanced Nursing. 2001;36(5):685-695.

4. Fink R, Thompson CJ, Bonnes D. Overcoming barriers and promoting the use of research in practice. Journal of Nursing Administration. 2005;35(3):121-129.

5. Yoder Wise P. The complex challenges of administrative research for the future. JONA. 2012;42(5):239-241. 